\title{
ASSESSMENT OF THE DEPENDENCE OF THE CLINICAL MANIFESTATION OF ACUTE GASTROENTERITIS CAUSED BY ROTAVIRUS ON ITS GENOTYPES
}

\author{
Mariia Teslenko \\ Paediatric Department ${ }^{1}$ \\ Infection Department \\ Kyiv City Children Clinical Hospital No 1. \\ 30 Bohatyrska str., Kyiv, 02000 \\ marydoc@ukr.net \\ Lyudmila Chernyshova \\ Paediatric Department ${ }^{1}$ \\ chernyshova@ukr.net \\ ${ }^{1}$ Shupyk National Medical Academy of Postgraduate Education \\ 9 Dorogozhytska str., Kyiv, Ukraine, 04112
}

\begin{abstract}
The leading cause of acute gastroenteritis (AGE) in children is rotavirus. In different countries, different rotavirus genotypes prevail and are associated with different severity of disease.

The purpose of our study was to identify the distribution of rotavirus genotypes in Kyiv, Ukraine, and to determine the correlation between the genotypes and course of disease.

Materials and methods. 978 children under 5 years of age were examined with AGE symptoms and not vaccinated against rotavirus. Determination of rotavirus antigen and genotype were performed using the immunoenzymatic assay and real-time RT-PCR.

We assessed the demographics, clinical manifestations of AGE, the Vesikari scale AGE severity.

Results. The G4P[8] genotype prevailed in Ukraine during 2014-2018. The G1P[8] was the second most common. G9P8 was the third, the fourth place was shared by G2P[4] and G3P[8]. Fever, as a manifestation, was more pronounced in G1P[8] and G3P[9].

The highest number of vomiting episodes per day occurred in the G1P[8] and G4P[8]-related cases.

Maximum of diarrhea episodes per day was observed in genotypes G1P[8], G3P[8], G4P [8] and G9P[8].

Mucus and blood in stool were found in genotypes G3P[8] (1/33.33 \%), G4P[8] - blood (1/2.27 \%).

The children with genotypes G1P[8] and G4P[8] had catarrhal symptoms.

More cases of moderate and severe dehydration, occurred in the G4P[8].

The Vesikari scale analysis showed that only G1P[8] led to mild cases(3.57 \%). The most widespread genotypes, G1P[8] and G4P[8], led to a moderate illness in $14.29 \%$ and $13.56 \%$ cases, respectively, and to a severe illness in $82.14 \%$ and $86.44 \%$ cases, respectively.

Conclusions. G4P[8] was associated with the most severe disease due to more frequent and prolonged vomiting, febrile fever and bloody diarrhea. G1P[8] and G4P[8] were associated with catarrh.
\end{abstract}

Keywords: genotypes of rotavirus, rotavirus in children, acute gastroenteritis.

DOI: $10.21303 / 2504-5679.2020 .001446$

\section{Introduction}

Acute gastroenteritis (AGE) is one of the major culprits of pediatric mortality worldwide. According to Global Disease Burden, children under the age of 5 comprise a third of all those who died of diarrhea [1].

Diarrhea is the third leading cause of childhood mortality globally, behind only pneumonia and complications from premature birth [1]. There is a variety of data regarding the role of different causative agents in gastroenteritis. However, rotavirus is arguably the leader. Vaccination is the only effective prevention against rotavirus. To date, 107 countries have introduced vaccine prevention against rotavirus, 103 of which - at the national level [2].

In different parts of the world, different genotypes of rotavirus prevail. The genotype G1P[8] represents over $70 \%$ of cases of rotavirus infection in North America, Europe, and Australia, but only about $30 \%$ in South America and Asia and $23 \%$ in Africa [3]. Overall, four G types (G1, G2, 
G3, and G4) associated with $\mathrm{P}[8]$ or $\mathrm{P}[4]$ account for over $88 \%$ of the strains detected worldwide. The G9 type associated with $\mathrm{P}[8]$ or $\mathrm{P}[6]$ accounts for another $4.1 \%$ [3].

Mohamed NF Shaheen reviewed 19 articles from 2000 to 2017 on diarrhea cases in Egypt. He obtained the following data: like anywhere in the world, G1P[8] was the most commonly detected rotavirus genotype, followed by G2P[4] and G3P[8] [4]. The researcher noted that after the introduction of rotavirus vaccination, not only the number of gastroenteritis cases reduced, but also the proportion of the new serotypes increased [4].

In Moscow, the genotype G4P[8] accounted for $38.7 \%$ of all rotavirus-positive cases between 2009 and 2014, being the prevailing genotype. Other genotypes, such as G1P[8], G9P[8], $\mathrm{G} 3 \mathrm{P}[8]$, and $\mathrm{G} 2 \mathrm{P}[4]$, occurred in $11.8 \%, 6.6 \%, 4.2 \%$, and $3.3 \%$ of cases respectively [5].

In almost all of the Latin America, the genotype G2P[4] prevails, except for Chile and Bolivia, where genotype G9P[8] is more common, occurring in $81.7 \%$ and $41.8 \%$ of the cases, respectively [6].

In Ukraine, rotavirus genotypes have been studied since 2006 as part of the World Health Organization Global Rotavirus Surveillance. In 2007-2008, two genotypes dominated - G1P[8] and G3P[8]; in 2008-2010, GM (mix) P[8] took the lead. Since 2011, the G4P[8] has been the most commonly detected [7].

The researchers' thoughts about the association between severity of condition and rotavirus genotype vary significantly $[8,9]$. The majority of researchers discover the dependence of the genotype on the severity of illness. However, the identical genotypes in the different parts of the world are associated with the different severity of disease. The researches, which were held in India and in England, the leading genotype was G9 and also it was a cause of the most severe disease [8, 10], but in the USA and Indonesia it was contrariwise, the disease, caused by G9, was not as severe as in the cases, caused by G2 [11, 12].

The aim of our study was to identify the distribution of genotypes in Kyiv, Ukraine, and to determine if there is a correlation between the genotypes of rotavirus currently circulating in Ukraine and severity and clinical manifestations of the disease.

\section{Materials and methods}

A total of 978 children under 5 years of age with symptoms of acute gastroenteritis were examined and hospitalized in the infectious disease isolation wards of Kyiv City Children Clinical Hospital No 1 between 2014 and 2018.

Inclusion criteria: children under 5 years of age with symptoms of acute gastroenteritis and not vaccinated against rotavirus infection, who required hospitalization and agreed to participate in the study.

Exclusion criteria: children over 5 years of age, children under 5 years of age, who did not require hospitalization, and children, whose parents (or legal representatives) refused to participate in the study. The study was conducted in accordance with the requirements of good clinical practice, the Council of Europe Convention on Human Rights and Biomedicine, the Helsinki Declaration of the World Medical Association and approved by the local ethics committee of the Shupyk National Medical Academy Of Postgraduate Education (protocol No. 9, from 28.11.2017).

A parents or legal representative of the child signed an informed consent to participate in a study. After consent was obtained, the children were included in the study and stool samples were taken.

A stool sample of $5 \mathrm{ml}$ was taken into a special container no later than 48 hours after admission to the hospital. Immediately after taking the stool sample, the container was placed in the refrigerator. Transportation to the laboratory took place in a refrigerated container.

Determination of the rotavirus antigen was carried out at the National Virus Reference Laboratory of the Center for Public Health using the immunoenzymatic assay (ELISA).

Random rotavirus-positive samples were tested for genotype determination in real-time by RT-PCR at the WHO reference laboratory (Republican Research and Practical Center for Epidemiology and Microbiology, Minsk, Belarus).

We assessed and compared the demographic data (age), clinical manifestations of acute gastroenteritis, severity of acute gastroenteritis on the Vesikari scale, conducted a survey on the 
outpatient course of the disease before admission to hospital, and collected catamnesis within 1 month after discharge from hospital.

We estimated the following clinical parameters: temperature at admission, maximum temperature, and duration of fever; number of vomiting episodes per day, number of repeated vomiting episodes per day during hospitalization after administration of antiemetic medications; number of diarrhea episodes per day before admission, duration of diarrhea prior to admission, number of diarrhea episodes per day during hospitalization, total duration of hospitalization, total duration of diarrhea, degree of dehydration, and severity of the disease on the Vesikari scale; frequency of signs such as bloating and abdominal pain, need for surgical consultation and surgical treatment, seizures, altered consciousness, runny nose, yawning, coughing and otitis, as well as stool consistency and the presence of pathological impurities in the stool.

The Vesikari severity scoring scale assesses the following parameters: the maximum number of diarrhea and vomiting episodes per 24 hours, duration of diarrhea and vomiting (days), temperature, degree of dehydration, and need for hospitalization. Every parameter is assessed on a scale from 1 to 3 . A total score of $<7$ corresponds to mild, $7-10$ - to moderate, and $\geq 11$ - to severe acute gastroenteritis. The maximum possible score is 20 [13].

Statistical analysis was performed with the STATA program. Chi-squared test $\left(\chi^{2}\right)$ was used for comparison of qualitative data. The quantitative data in this study did not follow normal distribution and, therefore, non-parametric criteria (median, interquartile range $(25 ; 75)$ ) were used.

\section{Results}

We examined 978 patients with symptoms of acute gastroenteritis. Rotavirus infection was diagnosed in 458 patients (46.83\%).

Of all the rotavirus-positive stool samples, 168 were randomly selected to be tested by RT-PCR for determining rotavirus genotypes.

Fig. 1 shows the distribution of genotypes identified in Kyiv in 2014-2018.

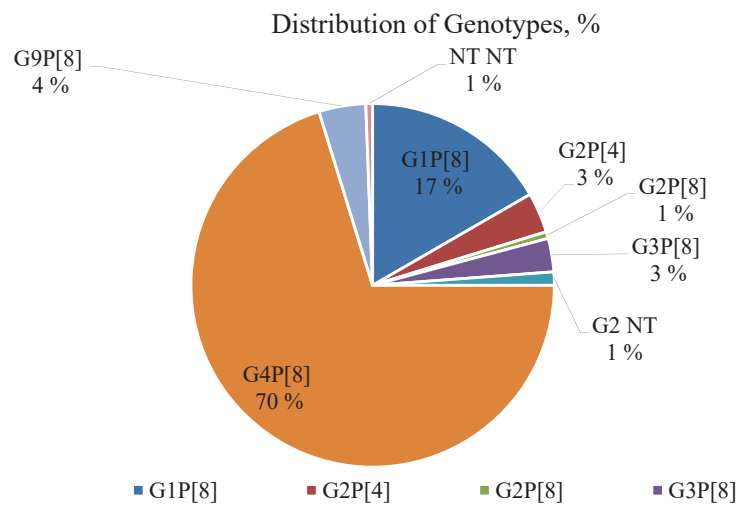

Fig. 1. Distribution of genotypes of rotavirus in 2014-2018, Kyiv

As seen from Fig. 1, the G4P[8] genotype dominated in Ukraine during 2014-2018.

The genotype G1P[8] was second most common in Ukraine. G9P[8] was the third commonest, while the fourth spot was shared by G2P[4] and G3P[8].

Among the studied stool samples, we detected cases of mixed infection with viral and bacterial pathogens. Among the identified genotypes, mixed infection was typical only for G4P[8] and detected in 2,99\%. Mixed infection with salmonella occurred in $1.19 \%$ of cases, and campylobacter, norovirus, and adenovirus in $0.60 \%$ of cases each.

We analyzed the peculiarities of clinical manifestations of various genotypes of rotavirus, which were widespread in Ukraine, specifically in Kyiv, in 2014-2018.

The age characteristics of the affected children were as follows.

For G1P[8] genotype, the average age was 27.9 (13.88; 38.47) months, for G2P[4] $11.95(9 ; 21)$ months, for G2P[8] - 11 months, for G2NT - $20.6(4 ; 37.2)$ months, for G3P[8] - 
$13.08(10 ; 20)$ months, for G4P[8] - $21.07(12 ; 35)$ months, for G9P[8] - $28(11 ; 31)$ months, for NT -1 month. The age difference was not statistically significant $(p>0.05)$.

The peculiarities of the disease onset and its further course with respect to the genotype are presented in Table 1.

Table 1

Clinical parameters in the compared groups, represented by qualitative data

\begin{tabular}{|c|c|c|c|c|c|c|c|c|c|}
\hline \multirow{2}{*}{ Symptom } & \multicolumn{8}{|c|}{ Rotavirus genotype, (Me (IQ 25;75)) } & \multirow{2}{*}{$P$-value } \\
\hline & G1P[8] & G2 NT & G2P $[4]$ & G2P[8] & G3P[8] & G4P[8] & G9P[8] & NT & \\
\hline Temperature at admission, ${ }^{\circ} \mathrm{C}$ & $\begin{array}{c}38.6 \\
(37.8 ; 39)\end{array}$ & $\begin{array}{c}37.65 \\
(37.5 ; 37.8)\end{array}$ & $\begin{array}{c}37.8 \\
(36.6 ; 38)\end{array}$ & 38.2 & $\begin{array}{c}37.8 \\
(37.7 ; 39.2)\end{array}$ & $\begin{array}{c}38 \\
(37.6 ; 38.6)\end{array}$ & $\begin{array}{c}37.7 \\
(37.6 ; 38.4)\end{array}$ & 38.1 & $p>0.05$ \\
\hline Temperature, duration before admission & $1(1 ; 1)$ & 0 & 2 & 0 & 1 & 1 & $1.5(1 ; 2)$ & 0 & $p>0.05$ \\
\hline $\begin{array}{l}\text { Maximum temperature during } \\
\text { hospitalization }\end{array}$ & $\begin{array}{c}38.6 \\
(37.8 ; 39)\end{array}$ & 36.6 & $\begin{array}{c}38.6 \\
(36.6 ; 39.2)\end{array}$ & 39.2 & $\begin{array}{c}37.5 \\
(37.2 ; 39.2)\end{array}$ & $\begin{array}{c}38.35 \\
(37.6 ; 38.8)\end{array}$ & $\begin{array}{c}37.6 \\
(36.6 ; 38.4)\end{array}$ & 38 & $p>0.05$ \\
\hline Total duration of fever & $4(3 ; 5)$ & $2(2 ; 2)$ & $3.5(3 ; 4)$ & 4 & $3(3 ; 4)$ & $3(3 ; 4.5)$ & $3(2 ; 6)$ & 4 & $p>0.05$ \\
\hline Number of vomiting episodes per day & $5(4 ; 6)$ & 2 & $4.5(3 ; 6)$ & 3 & $4(2 ; 8)$ & $5(3 ; 6)$ & $4.5(3 ; 6)$ & 3 & $p>0.05$ \\
\hline Vomiting duration & $2(1 ; 3)$ & $2.5(2 ; 3)$ & $2(2 ; 3)$ & $3(3 ; 3)$ & $2(2 ; 3)$ & $2(1 ; 3)$ & $3(2 ; 3)$ & $1(1 ; 1)$ & $p>0.05$ \\
\hline $\begin{array}{l}\text { Repeated vomiting during hospitaliza- } \\
\text { tion, number of episodes per day }\end{array}$ & $3(2 ; 3)$ & 0 & 2 & 0 & 1 & $2(1 ; 3)$ & 0 & 0 & $p>0.05$ \\
\hline Number of diarrhea episodes per day & $5(3 ; 6)$ & 0 & $4(1 ; 6)$ & 0 & 6 & $6.5(3 ; 10)$ & $6.5(3 ; 10)$ & 0 & $p>0.05$ \\
\hline Diarrhea duration before admission & $1(1 ; 1.5)$ & 0 & $2(1 ; 2)$ & 0 & 1 & $1(1 ; 2)$ & $2.5(2 ; 3)$ & 0 & $p>0.05$ \\
\hline $\begin{array}{l}\text { Number of diarrhea episodes per day } \\
\text { during hospitalization }\end{array}$ & $6(4.5 ; 10)$ & $6(5 ; 7)$ & $6(3 ; 6)$ & 7 & $7(6 ; 10)$ & $6(4 ; 10)$ & $7(4 ; 10)$ & 3 & $p>0.05$ \\
\hline Total diarrhea duration & $6(5.5 ; 6)$ & $5.5(5 ; 6)$ & $5(4 ; 9)$ & 11 & $6(4 ; 8)$ & $5(4 ; 7)$ & $7(4 ; 11)$ & 6 & $p>0.05$ \\
\hline Duration of hospitalization & $3(3 ; 4)$ & 2 & $3(2 ; 4)$ & 4 & $2(2 ; 2)$ & $2(2 ; 3)$ & $2(2 ; 4)$ & 1 & $p>0.05$ \\
\hline
\end{tabular}

As can be seen from Table 1, fever at the beginning of the disease was more pronounced in genotypes G1P[8] and G3P[9], and children were admitted to hospital from Day 1, while other genotypes were characterized by a gradual onset of fever. However, during hospitalization, the fever was high for almost all detected genotypes except for G2 NT. In G1P[8] genotype, the fever lasted the longest.

The genotypes G1P[8] and G4P[8] had the highest number of vomiting episodes per day.

The duration of vomiting was the shortest in the NT group; all other genotypes had the duration of vomiting of up to 3 days. However, the genotypes G1P[8], G2P [4], G3P [8], and G4P[8] had repeated vomiting episodes after administration of antiemetic medications during hospital stay.

We found that the greatest number of diarrhea episodes per day was observed in genotypes G1P[8], G3P[8], G4P[8], and G9P[8]. In genotypes G4P[8] and G9P[8], high frequency of diarrhea was observed from the very onset of the disease. The longest duration of diarrhea was observed in genotypes G9P[8] and G2P[8], but there were no differences in the duration of hospitalization between the genotypes.

We also evaluated the characteristics of stool consistency in different genotypes and the presence of pathological impurities. We obtained the following results. Watery stool was more typical of the genotypes G2P[4], G2P[8], G2 NT, while G1 P[8], G4P[8], and G9P[8] typically manifested with mushy stool $(p<0.05)$.

We have detected and analyzed the presence of pathological impurities such as blood and mucus in the stool. Pathological impurities were found in genotypes G3P[8] - mucus and blood (33.33\% (1)), G4P[8] - blood (2.27\% (1)), mucus (6.82\% (3)), G9P[8] - mucus (20\% (1)). We found no pathological impurities in the stool samples of children with other genotypes. 
The presence of pathological impurities in the stool was not associated with mixed infection. On the contrary, in cases where a child had a mixed infection with salmonella, campylobacter, adenovirus, or norovirus, this did not affect the consistency of the stool or the presence of blood in the stool. All cases with bloody diarrhea were classified as severe on the Vezikari scale.

Qualitative clinical manifestations, such as bloating, abdominal pain, need for surgical consultation and/or surgical treatment, seizures, altered consciousness, runny nose, yawning, coughing, and otitis are presented in Table 2.

Table 2 shows that children with acute rotavirus gastroenteritis with genotypes G4P[8] and G9P[8] were significantly more likely to require surgical consultation. Manifestations such as abdominal bloating and abdominal pain were more frequently observed with the genotype G9P[8]. We found that children with genotypes G1P[8] and G4P[8] had coughing and runny nose.

We evaluated the degree of dehydration with respect to the genotype of the rotavirus (Table 3 ).

Table 2

Clinical parameters in the compared groups, represented by qualitative data

\begin{tabular}{|c|c|c|c|c|c|c|}
\hline \multirow{2}{*}{ Symptoms } & \multicolumn{5}{|c|}{ Rotavirus genotype, \% (n) } & \multirow{2}{*}{$P$-value } \\
\hline & G1P[8] & G2P[4],[8], NT & G3P[8] & G4P[8] & G9P[8] & \\
\hline Bloating & $40(8)$ & $50(3)$ & $66.67(2)$ & $54.55(24)$ & $80(4)$ & $p>0.05$ \\
\hline Abdominal pain & $55(11)$ & $75(4)$ & $66.67(2)$ & $68,18(30)$ & $80(4)$ & $p>0.05$ \\
\hline Need for surgical consultation & 0 & 0 & 0 & $2,27(1)$ & $20(1)$ & $p<0.05$ \\
\hline Seizures & 0 & 0 & 0 & 0 & 0 & \\
\hline Refusal to eat & $100(20)$ & $100(6)$ & $100(3)$ & $97,73(43)$ & $100(5)$ & $p>0.05$ \\
\hline Reduced physical activity & $85(17)$ & $100(6)$ & $66,67(2)$ & $84.09(37)$ & $60(3)$ & $p<0.05$ \\
\hline Altered consciousness & 0 & 0 & 0 & 0 & 0 & \\
\hline Pharyngeal hyperemia & $60(12)$ & $50(3)$ & $66,67(2)$ & $59,09(26)$ & $40(2)$ & $p>0.05$ \\
\hline Coughing & $10(2)$ & 0 & $33,33(1)$ & $18,18(8)$ & 0 & $p>0.05$ \\
\hline Runny nose & $10(2)$ & 0 & 0 & $9,09(4)$ & 0 & $p>0.05$ \\
\hline Otitis & 0 & 0 & 0 & 0 & 0 & $p>0.05$ \\
\hline Total, $\mathrm{n}$ & 20 & 6 & 3 & 44 & 5 & 78 \\
\hline
\end{tabular}

Table 3

Degree of dehydration in compared groups

\begin{tabular}{lccccccc}
\hline \multirow{2}{*}{$\begin{array}{c}\text { Degree } \\
\text { of dehydration }\end{array}$} & G1P[8] & G2P[4],[8], NT & G3P[8] & G4P[8] & G9P[8] & NT NT & P-value \\
\cline { 2 - 6 } Mild & $53.57(15)$ & $44.44(4)$ & $40(2)$ & $28.81(34)$ & $57.14(4)$ & $100(1)$ & $p>0.05$ \\
Moderate & $32.14(9)$ & $44.44(4)$ & $60(3)$ & $44.92(53)$ & $42.86(3)$ & 0 & $p>0.05$ \\
Severe & $14.29(4)$ & $11.11(1)$ & 0 & $26.27(31)$ & 0 & 0 & $p>0.05$
\end{tabular}

As shown in Table 3, genotypes G1P[8] and G9P[8] accounted for the largest portion of cases with mild dehydration and received only oral rehydration solution. In the G4P[8] genotype, there were more cases of moderate and severe dehydration, which required infusion therapy.

In our study, all children with moderate and severe dehydration received oral rehydration solution along with infusion therapy. The infusion therapy for all groups lasted for one day.

To assess the severity of clinical manifestations of rotavirus gastroenteritis, the Vesikari scale was applied. 
Having analyzed the particularities of clinical manifestations for various genotypes on the Vesikari scale, we saw that only genotype G1P[8] led to mild cases (3.57\%), while all other genotypes led to moderate or severe disease. The genotypes that were the most widespread, G1P[8] and G4P[8], led to a moderate course of the disease in $14.29 \%$ (4) and $13.56 \%$ (16) cases, respectively, and to a severe course of the disease in $82.14 \%$ (23) and $86.44 \%$ (102) cases, respectively.

\section{Discussion}

Half of all AGEs in Kyiv were due to rotavirus infection. A slightly lower percentage (40.1\%) was reported by Russians in their 2009-2014 study [5]. Naturally, in countries where vaccination is mandatory, this percentage is much lower.

As described by Vesikari $\mathrm{T}$ and Matson DO, effectiveness of vaccination against rotavirus-associated hospitalization varies from 85 to $94 \%$, and against severe rotavirus diarrhea from $85 \%$ to $98 \%[13,14]$.

The dominated genotype in Kyiv during 2014-2018 was the same as prevailed in Moscow in $2009-2014$ [5].

The genotype G1P[8], the most prevalent genotype in Europe and North America[3], was the second most common in Ukraine.

Despite the fact that G9P[8], G2P[4] and G3P[8], were the most common genotypes in Latin America and China [15-17], in Ukraine these were the third and fourth commonest.

We investigated the frequency of mixed infections with bacteria and other viruses. In our study we received indicators less than in the world.

For example, Grimprel et al. conducted a systematic review of 173 journals published in English from 1989 to 2006 and found that the frequency of mixed infection ranged from $0.3 \%$ to $45.5 \%$ [18]. In Indonesia and Brazil, E. coli is the most common pathogen for mixed infection accompanying rotavirus; the incidence of co-infection reaches $42.5 \%$ [11] and $16.2 \%$, respectively [19]. In Kyiv, Ukraine the most common pathogen for co-infection was salmonella. Globally, the incidence of co-infection with salmonella varies from $0.5 \%$ to $4.8 \%$, with campylobacter from $1.0 \%$ to $3.2 \%$, with norovirus - from $2.4 \%$ to $23.5 \%$, and with adenovirus - from 1.0 to $8.8 \%$ [18]. The number of mixed infections of our study are the same only with salmonella.

Grimprel et al. reported, that the range of co-infections may reflect the local epidemiological situation, hygienic conditions, and economic development [18]. But we have not studied risk factors of co-infections, therefore we cannot say why these rates in Ukraine are low.

The age did not correlate with infection with specific genotypes.

When analyzing clinical manifestations, we found that vomiting was the most severe with genotypes G1P[8] and G4P[8] due to high frequency and repeating even after antiemetic drugs. All genotypes had the duration of vomiting of up to 3 days. In a study involving Indian children, the researchers found that the largest number of children in whom vomiting lasted longer than 3 days was in the G9 group (58.8\%). With the G12 type, vomiting was prolonged in $40.9 \%$ of cases, while with the G2 and G1 types vomiting lasted more than 3 days in a quarter of children $(26.3 \%$ and $24.7 \%$, respectively) [7]. Another study reported that children infected with the typeG2P[4] were older $(p=0.005)$ and presented with a more severe vomiting $(P=0.018,0.006)$ compared to children with G1P[8] [8]. Perhaps duration of vomiting in our study was shorter due to antiemetic drag administration. In the studies we analyzed, there were no indications for using antiemetics.

We found that the greatest number of diarrhea episodes per day and the longest duration of diarrhea was observed in genotypes G9P[8] unlike scientists from India [7]. Tarun Saluja et al. reported that genotype G1 was associated with the greatest number of diarrhea episodes per day and the longest duration of diarrhea in Indian children under 5 years old [7].

Despite the literature suggesting that rotavirus infection is not typically characterized by bloody diarrhea due to limited inflammatory response [11, 20, 21], we found pathological impurities such, es mucus and blood, in genotypes G3P[8], G4P[8].

It is known that rotavirus can have extraintestinal sites of infection and cause the corresponding symptoms [22]. In our study, we investigated the neurological status of the patients, as 
there were reports of rotavirus RNA discovered in the cerebrospinal fluid of some children with meningitis, encephalopathy, and encephalitis [22]. None of the participants of our study had seizures or impaired consciousness. But we found that children with genotypes G1P[8] and G4P[8] had coughing and runny nose. In a study conducted in Indonesia, the incidence of coughing in children with rotavirus infection reached $28.4 \%$, but the authors did not investigate the incidence of coughing for each genotype separately.

When we assessed dehydration degrees, we also saw a difference with other studies. We found that genotype G9P[8] accounted for mild dehydration and children received only oral rehydration solution. In contrast, in a study carried out in London, children with the G9 genotype were more likely to require intravenous rehydration [10]. In another study, genotypes G12 and G9 were accompanied by severe dehydration, and infusion rehydration therapy was used more frequently [7]. In our research severe dehydration associated firstly with G4 and after with G1 and G2.

We analyzed the clinical manifestations for various genotypes on the Vesikari scale, and we saw that only genotype G1P[8] led to mild cases. For example, in Indonesia mild cases were observed with genotype G2 and in the USA with G9 [9,23]. All genotypes, which we studied, led to moderate or severe disease. But the most severe disease was observed with genotype G4P[8], which was prevaluable. In the same time in India the most severe disease on the Vesikari scale was observed with G9, while in Indonesia this was with G2P[4], and in the USA - with G1, G2, and G3 [7, 9, 23].

Study limitations. The limitation of the study was the absence of outpatients, so we were not able to take into account patients who carried the disease at home and apparently had a mild or moderate course. In addition, some genotypes accounted for very few patients and therefore it is difficult to assess whether these or those clinical manifestations were typical for them.

Prospects for the further research. Investigate the severity of rotavirus AGE depending on the genotype in outpatient setting. Investigate the features of clinical manifestations of rare genotypes.

\section{Conclusions}

1. In Kyiv, G4P[8] was associated with the most severe course of the disease due to more frequent and prolonged vomiting, prolonged febrile fever and bloody diarrhea (in $2.27 \%$ of patients).

2. G1P[8] and G4P[8], unlike in other genotypes, were associated with characteristic catarrhal symptoms.

3. Genotype G1P[8] in non-vaccinated children induced mild disease in $4 \%$ of cases.

\section{Conflict of interests}

The authors declare that they have no conflicts of interest.

\section{References}

[1] Dadonaite, B., Ritchie, H., Roser, M. (2019). Diarrheal diseases. Our World Data. Available at: https://ourworldindata.org/ diarrheal-diseases

[2] Global Introduction Status (2020). Rota Council. Available at: https://rotacouncil.org/vaccine-introduction/global-introduction-status/

[3] Santos, N., Hoshino, Y. (2004). Global distribution of rotavirus serotypes/genotypes and its implication for the development and implementation of an effective rotavirus vaccine. Reviews in Medical Virology, 15 (1), 29-56. doi: http://doi.org/10.1002/rmv.448

[4] Shaheen, M. N. (2018). Burden of Adenovirus, Astrovirus, Norovirus and Rotavirus Gastroenteritis in Egyptian Children during 2000-2017. Journal of Medical Microbiology Diagnosis, 7 (3). doi: http://doi.org/10.4172/2161-0703.1000283

[5] Kiseleva, V., Faizuloev, E., Meskina, E., Marova, A., Oksanich, A., Samartseva, T. et. al. (2018). Molecular-Genetic Characterization of Human Rotavirus A Strains Circulating in Moscow, Russia (2009-2014). Virologica Sinica, 33 (4), $304-313$. doi: http://doi.org/10.1007/s12250-018-0043-0

[6] Santos, V. S., Marques, D. P., Martins-Filho, P. R. S., Cuevas, L. E., Gurgel, R. Q. (2016). Effectiveness of rotavirus vaccines against rotavirus infection and hospitalization in Latin America: systematic review and meta-analysis. Infectious Diseases of Poverty, 5 (1). doi: http://doi.org/10.1186/s40249-016-0173-2

[7] Chernyshova, L. I., Radionova, N. M., Demchyshyna, I. V., Kotlik, L. S., Sadkova, O. B., Samoilovich, E. O. et. al. (2018). Observations on the epidemiology of rotavirus infection among hospitalized children younger than 5 years in 2 Ukrainian hospitals, 2007-2015. Vaccine, 36 (51), 7798-7804. doi: http://doi.org/10.1016/j.vaccine.2017.11.044 
[8] Saluja, T., Dhingra, M. S., Sharma, S. D., Gupta, M., Kundu, R., Kar, S. et. al. (2016). Association of rotavirus strains and severity of gastroenteritis in Indian children. Human Vaccines \& Immunotherapeutics, 13 (3), 711-716. doi: http://doi.org/ $10.1080 / 21645515.2016 .1238994$

[9] Shim, J. O., Chang, J. Y., Shin, S., Moon, J. S., Ko, J. S. (2016). Changing distribution of age, clinical severity, and genotypes of rotavirus gastroenteritis in hospitalized children after the introduction of vaccination: a single center study in Seoul between 2011 and 2014. BMC Infectious Diseases, 16 (1). doi: http://doi.org/10.1186/s12879-016-1623-y

[10] Cubitt, W. D., Steele, A. D., Iturriza, M. (2000). Characterisation of rotaviruses from children treated at a London hospital during 1996: emergence of strains G9P2A[6] and G3P2A[6]. Journal of Medical Virology, 61 (1), 150-154. doi: http://doi.org/ 10.1002/(sici)1096-9071(200005)61:1<150::aid-jmv24 > 3.0.co;2-w

[11] Clark, H. F., Lawley, D. A., Schaffer, A., Patacsil, J. M., Marcello, A. E., Glass, R. I. et. al. (2004). Assessment of the Epidemic Potential of a New Strain of Rotavirus Associated with the Novel G9 Serotype Which Caused an Outbreak in the United States for the First Time in the 1995-1996 Season. Journal of Clinical Microbiology, 42 (4), 1434-1438. doi: http://doi.org/10.1128/ jcm.42.4.1434-1438.2004

[12] Sudarmo, S., Shigemura, K., Athiyyah, A., Osawa, K., Wardana, O., Darma, A. et. al. (2015). Genotyping and clinical factors in pediatric diarrhea caused by rotaviruses: one-year surveillance in Surabaya, Indonesia. Gut Pathogens, 7 (1), 3. doi: http:// doi.org/10.1186/s13099-015-0048-2

[13] Ruuska, T., Vesikari, T. (1990). Rotavirus Disease in Finnish Children: Use of Numerical Scores for Clinical Severity of Diarrhoeal Episodes. Scandinavian Journal of Infectious Diseases, 22 (3), 259-267. doi: http://doi.org/10.3109/00365549009027046

[14] Vesikari, T., Matson, D. O., Dennehy, P., Van Damme, P., Santosham, M., Rodriguez, Z. (2006). Safety and Efficacy of a Pentavalent Human-Bovine (WC3) Reassortant Rotavirus Vaccine. New England Journal of Medicine, 354 (1), 23-33. doi: http:// doi.org/10.1056/nejmoa052664

[15] Ruiz-Palacios, G. M., Pérez-Schael, I., Velázquez, F. R., Abate, H., Breuer, T., Clemens, S. C. et. al. (2006). Safety and Efficacy of an Attenuated Vaccine against Severe Rotavirus Gastroenteritis. New England Journal of Medicine, 354 (1), 11-22. doi: http://doi.org/10.1056/nejmoa052434

[16] Tian, Y., Chughtai, A. A., Gao, Z., Yan, H., Chen, Y., Liu, B. et. al. (2018). Prevalence and genotypes of group A rotavirus among outpatient children under five years old with diarrhea in Beijing, China, 2011-2016. BMC Infectious Diseases, 18 (1). doi: http://doi.org/10.1186/s12879-018-3411-3

[17] Chernyshova, L. I., Teslenko, M. Y., Radionova, N. M., Demchishina, I. V., Kotlik, L. V., Sadkova, O. B. et. al. (2017). Longitudinal Observation on Rotavirus Infection in Children Aged under 5 Years Old Hospitalized in 2 Hospitals of Ukraine in 2006-2015. Child`s health, 7 (75), 117-123. doi: http://doi.org/10.22141/2224-0551.7.75.2016.86736

[18] Grimprel, E., Rodrigo, C., Desselberger, U. (2008). Rotavirus Disease: Impact of Coinfections. The Pediatric Infectious Disease Journal, 27, 3-10. doi: http://doi.org/10.1097/inf.0b013e31815eedfa

[19] Souza, E. C., Martinez, M. B., Taddei, C. R., Mukai, L., Gilio, A. E., Racz, M. L. et. al. (2002). Perfil etiológico das diarréias agudas de crianças atendidas em São Paulo. Jornal de Pediatria, 78 (1). doi: http://doi.org/10.1590/s0021-75572002000100008

[20] Morris, A. P., Estes, M. K. (2001). Microbes and microbial toxins: paradigms for microbial-mucosal interactions. VIII. Pathological consequences of rotavirus infection and its enterotoxin. American Journal of Physiology-Gastrointestinal and Liver Physiology, 281 (2), G303-G310. doi: http://doi.org/10.1152/ajpgi.2001.281.2.g303

[21] Lundgren, O., Svensson, L. (2001). Pathogenesis of Rotavirus diarrhea. Microbes and Infection, 3 (13), 1145-1156. doi: http:// doi.org/10.1016/s1286-4579(01)01475-7

[22] Crawford, S. E., Ramani, S., Tate, J. E., Parashar, U. D., Svensson, L., Hagbom, M. et. al. (2017). Rotavirus infection. Nature Reviews Disease Primers, 3 (1). doi: http://doi.org/10.1038/nrdp.2017.83

[23] Wardana, O. P., Athiyyah, A. F., Budiono, Soedirham, O., Soegijanto, S. (2015). The Role of Rotavirus Genotype in the Severity of Acute Diarrhea in Children Under 5 Years Old at Surabaya, Indonesia. Journal of Human Virology \& Retrovirology, 2 (2). doi: http://doi.org/10.15406/jhvrv.2015.02.00039 